\title{
Genome-Wide Identification and Evaluation of Reference Genes for Quantitative RT-PCR Analysis during Tomato Fruit Development
}

OPEN ACCESS

Edited by:

Hinanit Koltai,

Agricultural Research Organization,

Israel

Reviewed by:

Panagiotis Kalaitzis,

Mediterranean Agronomic Institute of

Chania, Greece

Antonio Ferrante,

Università degli Studi di Milano, Italy

*Correspondence:

Hongjian Wan

wanhongjian@sina.com

${ }^{\dagger}$ These authors have contributed equally to this work

Specialty section

This article was submitted to Crop Science and Horticulture,

a section of the journal

Frontiers in Plant Science

Received: 19 April 2017 Accepted: 03 August 2017

Published: 29 August 2017

Citation:

Cheng Y, Bian W, Pang X, Yu J, Ahammed GJ, Zhou G, Wang $R$, Ruan M, Li Z, Ye Q, Yao Z, Yang Y and

Wan H (2017) Genome-Wide

Identification and Evaluation of

Reference Genes for Quantitative RT-PCR Analysis during Tomato Fruit

Development.

Front. Plant Sci. 8:1440

do: 10.3389/fpls.2017.01440

\author{
Yuan Cheng ${ }^{1 \dagger}$, Wuying Bian ${ }^{2 \dagger}$, Xin Pang ${ }^{3}$, Jiahong Yu ${ }^{1}$, Golam J. Ahammed ${ }^{4}$, \\ Guozhi Zhou ${ }^{1}$, Rongqing Wang ${ }^{1}$, Meiying Ruan ${ }^{1}$, Zhimiao Li ${ }^{1}$, Qingjing Ye ${ }^{1}$, Zhuping Yao ${ }^{1}$, \\ Yuejian Yang ${ }^{1}$ and Hongjian Wan ${ }^{1 *}$ \\ ' State Key Laboratory Breeding Base for Zhejiang Sustainable Pest and Disease Control, Institute of Vegetables, Zhejiang \\ Academy of Agricultural Sciences, Hangzhou, China, ${ }^{2}$ Zhejiang Agricultural Technology Extension Center, Hangzhou, China, \\ ${ }^{3}$ Suzhou Polytechnic Institute of Agriculture, Suzhou, China, ${ }^{4}$ Department of Horticulture, Zhejiang University, Hangzhou, \\ China
}

Gene expression analysis in tomato fruit has drawn increasing attention nowadays. Quantitative real-time PCR ( $\mathrm{PPCR}$ ) is a routine technique for gene expression analysis. In qPCR operation, reliability of results largely depends on the choice of appropriate reference genes (RGs). Although tomato is a model for fruit biology study, few RGs for QPCR analysis in tomato fruit had yet been developed. In this study, we initially identified 38 most stably expressed genes based on tomato transcriptome data set, and their expression stabilities were further determined in a set of tomato fruit samples of four different fruit developmental stages (Immature, mature green, breaker, mature red) using qPCR analysis. Two statistical algorithms, geNorm and Normfinder, concordantly determined the superiority of these identified putative RGs. Notably, SIFRG05 (Solyc01g104170), SIFRG12 (Solyc04g009770), SIFRG16 (Solyc10g081190), SIFRG27 (Solyc06g007510), and SIFRG37 (Solyc11g005330) were proved to be suitable RGs for tomato fruit development study. Further analysis using geNorm indicate that the combined use of SIFRG03 (Solyc02g063070) and SIFRG27 would provide more reliable normalization results in GPCR experiments. The identified RGs in this study will be beneficial for future qPCR analysis of tomato fruit developmental study, as well as for the potential identification of optimal normalization controls in other plant species.

Keywords: qPCR analysis, normalization, reference gene (RG), tomato, fruit development

\section{INTRODUCTION}

Tomato (Solanum lycopersicum) is an economically important horticultural crop in terms of production, flavor, and nutritional value of fruits. During the course of development and ripening, tomato fruits undergo a number of physiological and biochemical processes that bring forth the overall changes in fruit size, color, texture, and aroma (Klee and Giovannoni, 2011; Ruiz-May and Rose, 2012). Moreover, tomato is considered as an important model for genetic and molecular studies, partly due to its typical climacteric fruit property (Colombiet et al., 2016). A number of studies had been carried out to improve agronomic traits of tomato fruits, including size, pigment content, and flavor substances focusing on the metabolic and regulatory networks (Klee and Giovannoni, 2011; Ruiz-May and Rose, 2012; Tieman et al., 2017). Recent developments in genomic 
resources and bioinformatics tools (e.g., Genome-wide association study, GWAS) have enabled rapid elucidation of the complicated biological processes that occur during fruit development. Moreover, relative gene expression profiles during fruit development provide valuable clues for understanding the biological functions of the corresponding genes. So far, quantitative real-time PCR (qPCR) is considered as one of the most efficient tools for the measurement of transcript abundance of a gene due to its high accuracy, sensitivity, and reproducibility (Ginzinger, 2002; Bustin and Nolan, 2004; Gachon et al., 2004; Bustin et al., 2005).

In qPCR experiments, the reliability of the results predominantly depends on the appropriateness of RGs used for normalization, which should be stably expressed under the given experimental conditions (Pfaffl, 2004; Huggett et al., 2005). Highly stable expression of RGs could effectively remove non-biological variations including the difference in amounts, variability in enzymatic efficiency of reverse transcriptase, and sample differences in the overall transcriptional activity (Suzuki et al., 2000; Bustin et al., 2005; Huggett et al., 2005; Exposito-Rodriguez et al., 2008; Gutierrez et al., 2008). Generally speaking, an ideal RG should be a gene that is stably expressed under various experimental conditions or among different tissues (Czechowski et al., 2005; Huggett et al., 2005; ExpositoRodriguez et al., 2008; Dekkers et al., 2012; Wang et al., 2012). Housekeeping genes (HKGs) encoding, e.g., GAPDH, Actin, UBI, and 18 sRNAs, are usually regarded as suitable normalization controls (Stürzenbaum and Kille, 2001). However, some previous studies reported that the transcription of several HKGs can be fluctuated considerably under certain conditions (Czechowski et al., 2005; Jain et al., 2006; Gutierrez et al., 2008; Jian et al., 2008; Jarosova and Kundu, 2010), which illustrates the importance of systematic identification or validation of optimal RGs in order to avoid inaccurate results (Gutierrez et al., 2008; Guenin et al., 2009). In practice, the expression levels of most RGs are proved to be dependent on the specific conditions, including experimental treatments, tissue types, or developmental stages (Czechowski et al., 2005; Jian et al., 2008; Jarosova and Kundu, 2010). Hence, no single RG is widely applicable for different experimental conditions. Systematic evaluation of RGs must be conducted on each qPCR experiment before their use (Bustin et al., 2009; Jacob et al., 2013). Furthermore, it has been well-recognized that in some cases, one single RG may not be adequate for reliable normalization in gene expression analysis (Yoo et al., 2009; Cassan-Wang et al., 2012). To date, some common statistical algorithms, including geNorm (Vandesompele et al., 2002), NormFinder (Andersen et al., 2004), and Bestkeeper (Pfaffl, 2004), have been developed to determine the expression stabilities of RGs, which effectively simplify the selection of appropriate RGs for qPCR analysis.

Over the decades, a good number of stably expressed RGs have specifically been identified for normalization in the fruits of several fruit crops, such as papaya (Zhu et al., 2012), blueberry (Die and Rowland, 2013), and watermelon (Kong et al., 2015). For tomato, although suitable RGs have been identified under different experimental conditions including biotic/abiotic stresses (Løvdal and Lillo, 2009; Mascia et al., 2010) and various tissues of different developmental stages (Suzuki et al., 2000; Dekkers et al., 2012), very limited number of RGs in tomato fruit have been characterized so far (Coker and Davies, 2003; Baldassarre et al., 2015). Moreover, we noticed that most studies involving RG identification, including those relevant to tomato fruit developmental studies, were mainly based on the evaluation or validation of some already known candidate RGs (Mostly HKGs), which are convenient for implementation but also greatly limit the choice of best RGs. With the availability of tomato genome sequence and subsequent transcriptome data (SGN:Sol genomics network, https://solgenomics.net/; TFGD: Tomato functional genomics database,http://ted.bti.cornell.edu/), our study was aimed to identify some novel RGs for qPCR analysis of tomato fruit development within the entire genome level.

In this study, we initially evaluated the expression stabilities of all the tomato (S. lycopersicum L.) genes during various fruit developmental stages based on the RNA-seq data. A total of 38 novel genes stably expressed were identified as putative RGs and were further evaluated through qPCR analysis. Moreover, using two different statistical algorithms (geNorm and Normfinder), five optimal RGs were identified as optimal RGs for normalization during different stages of tomato fruit development. Furthermore, we also found that the combined use of two top-ranked RGs (SlFRG03 and SlFRG27) would potentially improve the accuracy of the qPCR results. Thus, based on the analysis of the entire tomato genome database, we comprehensively identified and evaluated the optimal RGs through large-scale biological information mining and qPCR techniques. These results not only provide useful RG resources for accurate gene expression studies in tomato fruit, but also shed light on the RG identification in fruit developmental study of other plant species.

\section{MATERIALS AND METHODS}

\section{Collection and Evaluation of the Previously Reported RGs}

Firstly, the potential tomato RGs reported in previous studies (Coker and Davies, 2003; Exposito-Rodriguez et al., 2008; Løvdal and Lillo, 2009; Mascia et al., 2010; Müller et al., 2015) were selected to evaluate the expression stabilities during different stages of tomato (S. lycopersicum L.) fruit development based on the RNA-seq data downloaded from the TFGD (http://ted.bti. cornell.edu/). Furthermore, the orthologous genes of 11 potential RGs for watermelon fruit development (Kong et al., 2015) were identified. All the potential RGs selected from RNA-seq data were evaluated for expression stability. Details including accession number, gene locus, gene description, and RNA-seq values were listed in Table 1 and Supplementary Table 1. The corresponding gene sequences of these candidate RGs were collected from NCBI (National Center for Biotechnology Information: https:// www.ncbi.nlm.nih.gov/), SGN (https://solgenomics.net/), TFGD (http://ted.bti.cornell.edu/) (Fraser et al., 1994), and CuGenDB (http://www.icugi.org/). Through Blastn search, the orthologous genes (E-value was set at $1 \mathrm{e}^{-5}$ ) were collected in tomato genome 
TABLE 1 | Previously reported RGs in tomato or watermelon.

\begin{tabular}{|c|c|c|c|c|}
\hline Gene symbol & Accession number & Gene locus & Gene description & Sequence identities (\%) \\
\hline GAPDH & U97257 & Solyc05g014470 & Glyceraldehyde 3-phosphate dehydrogenase & 99 \\
\hline EF1 $\alpha$ & X53043 & Solyc06g005060 & Elongation factor 1 -alpha & 98 \\
\hline DNAJ & AF124139 & Solyc11g006460 & DNAJ-like protein & 96 \\
\hline TUA & AC122540 & Solyc09g074220 & Musmusculus BAC clone & 99 \\
\hline $\mathrm{CHI9}$ & NM001247474 & Solyc10g055810 & Chitinase (CHI9) & 99 \\
\hline ACT & ВТ013707 & Solyc03go'78400 & Actin & 100 \\
\hline CYP & AK326854 & Solyc01g108340 & Cyclophilin Elongation factor $1-\alpha$ & 100 \\
\hline GAPDH & U93208 & Solyc03g111010 & Glyceraldehyde 3-phosphate dehydrogenase & 98 \\
\hline UBI & X58253 & Solyc01g056940 & Ubiquitin 3 & 99 \\
\hline TIP41 & SGN-U321250 & Solyc10g049850 & TIP41-like family protein & 99 \\
\hline SAND & SGN-U316474 & Solyc03g115810 & SAND family protein & 99 \\
\hline CAC & SGN-U314153 & Solyc08g006960 & Clathrin adaptor complexes medium subuit & 100 \\
\hline Expressed & SGN-U346908 & Solyc07g025390 & Expressed sequence & 98 \\
\hline PK & TC123837 & Solyc07g066610 & Phosphoglycerate kinase & 100 \\
\hline $\mathrm{CBCP}$ & TC115713 & Solyc01g028810 & Chaperonin-60 beta chain prec & 100 \\
\hline DNAJ & TC123959 & Solyc04g009770 & DNAJ-like protein & 100 \\
\hline AT5G & TC123964 & Solyc06g072120 & AT5g & 100 \\
\hline CICAC & Cla016178 & Solyc06g061150 & Clathrin adaptor complex subunit & 95 \\
\hline CIPP2A & Cla021905 & Solyc01g011340 & Protein phosphatase $2 \mathrm{~A}$ regulatory subunit $\mathrm{A}$ & 82 \\
\hline CIRAN & Cla012277 & Solyc05g023800 & A member of RAN GTPase gene family & 82 \\
\hline \multirow[t]{18}{*}{ Cl18SrRNA } & Cla010159 & Solyc05g007050 & 18SrRNA & 82 \\
\hline & & Solyc10g006100 & Transcription initiation factor TFIID subunit 6 & 100 \\
\hline & & Solyc07g062920 & Genomic DNA chromosome 5 TAC clone K19P17 & 100 \\
\hline & & Solyc01g111780 & Importin beta-2 subunit & 100 \\
\hline & & Solyc06g051420 & PHD finger family protein & 100 \\
\hline & & Solyc12g057120 & Subunit Vlb of cytochrome c oxidase & 100 \\
\hline & & Solyc01g009290 & Polyribonucleotide 5'-hydroxyl-kinase Clp1 & 100 \\
\hline & & Solyc09g018730 & Ubiquitin carboxyl-terminal hydrolase family 1 & 100 \\
\hline & & Solyc02g088110 & Polypyrimidine tract-binding protein-like & 100 \\
\hline & & Solyc08g060860 & P1 clone MSJ11 & 100 \\
\hline & & Solyc09g009640 & U6 snRNA-associated Sm-like protein LSm7 & 100 \\
\hline & & Solyc04g015370 & Acyl carrier protein & 100 \\
\hline & & Solyc08g005140 & Serine/threonine-protein kinase BUD32 & 100 \\
\hline & & Solyc02g062920 & Splicing factor U2AF large subunit & 100 \\
\hline & & Solyc10g076910 & ATP-dependent RNA helicase-like protein & 100 \\
\hline & & Solyc03g121980 & Developmentally-regulated GTP-binding protein 2 & 100 \\
\hline & & Solyc01g097140 & Dual-specificity tyrosine-phosphatase CDC25 & 100 \\
\hline & & Solyc07g007040 & Zinc finger CCCH-type protein & 100 \\
\hline
\end{tabular}


TABLE 1 | Continued

\begin{tabular}{|c|c|c|c|c|}
\hline Gene symbol & Accession number & Gene locus & Gene description & Sequence identities (\%) \\
\hline & & Solyc06g069310 & Nuclear transcription factor Y subunit B-6 & 100 \\
\hline & & Solyc03g078020 & Peptide chain release factor 1 & 100 \\
\hline & & Solyc02g089230 & DSBA oxidoreductase family protein & 100 \\
\hline & & Solyc06g036720 & HLA-B associated transcript 3 & 100 \\
\hline & & Solyc11g071930 & DnaJ homolog subfamily $\mathrm{C}$ member 8 & 100 \\
\hline & & Solyc06g084000 & Heterogeneous nuclear ribonucleoprotein $\mathrm{K}$ & 100 \\
\hline & & Solyc04g009230 & Mitosis protein dim1 & 100 \\
\hline & & Solyc06g073870 & DNA-directed RNA polymerase II subunit RPB4 & 100 \\
\hline & & Solyc09g055760 & T-snare & 100 \\
\hline & & Solyc10g005800 & CWC15 homolog & 100 \\
\hline & & Solyc12g021130 & 3-beta-hydroxysteroid dehydrogenase-like & 100 \\
\hline & & Solyc01g079330 & RNA helicase DEAD3 & 100 \\
\hline & & Solyc07g041550 & RNA polymerase-associated protein Ctr9 homolog & 100 \\
\hline & & Solyc03g059420 & Sister chromatid cohesion 2 & 100 \\
\hline & & Solyc11g071950 & Unknown & 100 \\
\hline & & Solyc12g099570 & Heat shock factor binding protein 2 & 100 \\
\hline & & Solyc10g044900 & CASTOR protein & 100 \\
\hline & & Solyc10g084270 & Importin alpha-2 subunit & 100 \\
\hline & & Solyc06g016750 & Transcription factor & 100 \\
\hline & & Solyc02g092380 & Peptidyl-prolylcis-trans isomerase & 100 \\
\hline
\end{tabular}

Detailed information was derived from NCBI (https://www.ncbi.nlm.nih.gov/), TFGD (https://www.ncbi.nlm.nih.gov/), CuGenDB (http://www.icugi.org/), and SGN (https://solgenomics. net/). The three redundant genes were labeled in bold.

databases (SGN) (Table 1). The Reads Per Kilobase Million (RPKM) value of each reported RG was collected from RNA-seq data, and the average expression (AVE) and standard deviation $(S D)$ values in different fruit developmental stages of fruit were calculated (Supplementary Table 1; Table 1). Relative expression level per gene was calculated through dividing the expression value of each fruit development stage by AVE (Figure 1A). $\mathrm{CV}$ (co-efficient variation) value of each gene during fruit development was calculated as the ratio of the $S D$ to the AVE (Supplementary Table 3).

\section{Identification of Stably Expressed Genes through Mining Fruit RNA-Seq Data Set}

Through mining the entire set of RNA-seq data file (TFGD), genes with medium expression $(200<\mathrm{RPKM}$ value $<$
2,000) at all stages of fruit development were selected (Supplementary Table 2). The CV value of each gene was calculated (Supplementary Table 2) and the genes with CV < 0.35 were chosen as putative RGs in the following qPCR analysis (Table 2; Supplementary Table 3).

\section{Plant Materials}

The tomato (S. lycopersicum L.) inbred line "S14" was used in the current experiments. Generally, the fruits of this genotype become completely mature (red flash) 45 days after pollination. On month old tomato seedlings were transplanted to the greenhouse at Zhejiang Academy of Agricultural Sciences, Hangzhou, China (east longitude $120^{\circ} 2^{\prime \prime}$, north latitude $30^{\circ} 27^{\prime \prime}$ ) on August 5th (late summer), 2016. Field management was implemented following the standard commercial practices. Tomato fruits were harvested at four developmental stages 

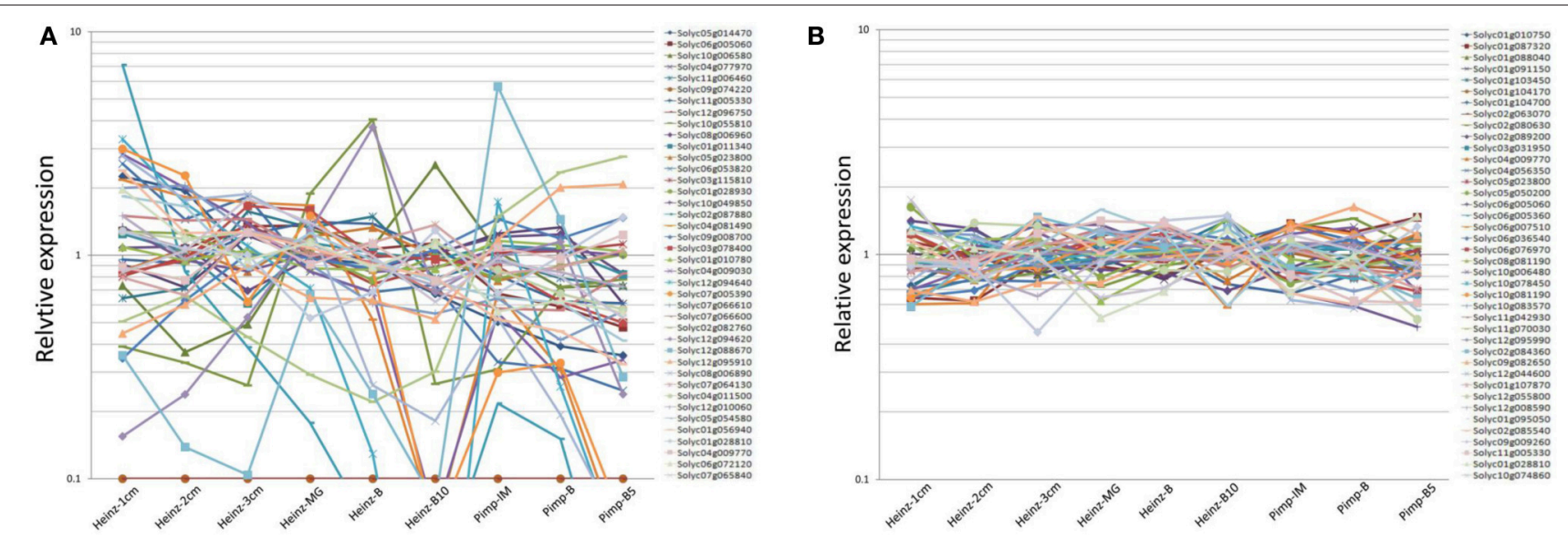

FIGURE 1 | Relative expression analysis of previously reported RGs (A) and newly identified RGs (B) over different tomato fruit developmental stages. Expression levels (RPKM values) were derived from RNA seq dataset (http://ted.bti.cornell.edu/). Relative expression level per gene were calculated by dividing the expression value (RPKM value) by the average expression level across various developmental stages of tomato fruits (Heinz-1 cm, Heinz-2 cm, Heinz-3 cm, Heinz-MG, Heinz-B, Heinz-MG, Heinz-B10, Pimp-IM, Pimp-B, and Pimp-B5) (Supplementary Tables 1-3).

(Immature: IM, Mature Green: MG, Breaker: B, and Mature Red: MR) from October to November (autumn), 2016. Three fruits were randomly collected at each sampling point, with each of them representing a biological replicate. All the samples were frozen in liquid nitrogen and stored at $-80^{\circ} \mathrm{C}$ for subsequent experiments.

\section{Total RNA Isolation and cDNA Synthesis}

Total RNA from collected samples was isolated using TRIZOL reagent according to the manufacturer's protocol (Tiangen, Beijing, China) as described previously(Cheng et al., 2016). The concentration and purity of extracted RNA were measured using a BioDropULite spectrophotometer (Biochrom, England), and RNA samples with A260/A280>1.8 and A260/A230>2.0 (indicating good RNA quality) were used for following experiments. All RNA samples were adjusted to the same concentration in order to ensure that the RNA input was homogenized for subsequent reverse transcription reactions using a mix of random primers. Then, according to the manufacturer's instruction (TIANGEN, Beijing, China), genomic DNAs (gDNA) were eliminated from the RNA samples and single-stranded cDNAs were synthesized.

\section{Primer Design and qPCR Analysis}

Gene-specific primers were designed using Real-time PCR (TaqMan) primer design (https://www.genscript.com/) as listed in Table 2. The qPCR experiments was performed in a 96-well plate using SYBR Green-based PCR assay. A $20 \mu \mathrm{L}$ reaction mixture [ $6 \mu \mathrm{L}$ diluted cDNA (10 ng), $10 \mu \mathrm{L}$ SYBR Green PCR Master Mix (Invitrogen, USA), $250 \mathrm{nM}$ of each primer and, 0.1 $\mu \mathrm{L}$ ROX] was subjected to the following procedure: $10 \mathrm{~min}$ at $94^{\circ} \mathrm{C}, 30$ cycles of $45 \mathrm{~s}$ at $94^{\circ} \mathrm{C}, 45 \mathrm{~s}$ at $55^{\circ} \mathrm{C}$, and $1 \mathrm{~min}$ at $72^{\circ} \mathrm{C}$ following a $7 \mathrm{~min}$ extension at $72^{\circ} \mathrm{C}$ ( $\mathrm{ABI}$ real time PCR system, USA). Three technical duplications performed for all of the RGs. The amplified products were resolved by $1.5 \%$ agarose gel electrophoresis (Supplementary Figure 1). The melting curves were created and exhibited for all the investigated qPCR products in the qPCR experiments (Supplementary Figure 2).

\section{Evaluation of RG Expression Stability Using geNorm and Normfinder}

The expression levels of the detected genes were obtained through the qPCR analysis and the results were demonstrated as Ct values (Supplementary Table 4). The amplification efficiency (E) and correlation coefficient $\left(R^{2}\right)$ for each gene were calculated using the standard curve method by amplifying the 10 -fold serial dilution of cDNA samples. The amplification efficiency (E) was

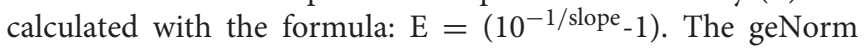
and NormFinder software packages were used to evaluate the gene expression stability as described before in this study (Vandesompele et al., 2002; Andersen et al., 2004). The geNorm applet not only provides a measure of gene expression stability value $(\mathrm{M})$, but also creates pairwise variation values $(\mathrm{V})$ to determine the minimum number of RGs required for reliable normalization, no additional genes were required when the pairwise variation $(\mathrm{Vn} / \mathrm{n}+1)$ was lower than 0.15 (Vandesompele et al., 2002). The NormFinder measured the variations across groups and determine the expression stability of each tested gene (Andersen et al., 2004). Lower stability values (M) in both geNorm and Normfinder implied the higher expression stability of the genes. The stability values (M) obtained from geNorm and NormFinder were listed in Supplementary Table 5.

\section{RESULTS}

\section{Evaluation of Previously Reported RGs during Tomato Fruit Development}

In our study, tomato (S. lycopersicum L.) genome database in the SGN (https://solgenomics.net/) and transcriptome data derived from the TFGD (http://ted.bti.cornell.edu/) were used for 
TABLE 2 | Description of the newly identified RGs, primer sequences, and amplification characteristics.

\begin{tabular}{|c|c|c|c|c|c|c|}
\hline Gene ID & Gene locus $^{a}$ & Gene description & Primer sequence $\left(5^{\prime}-3^{\prime}\right)$ & Product size (bp) & $\mathbf{E}$ & $R^{2}$ \\
\hline \multirow[t]{2}{*}{ SIFRG01 } & Solyc06g005360 & Actin depolymerizing factor 3 & F: TGACAAAGGAAAGTGTCCCA & 133 & 0.76 & 0.994 \\
\hline & & & R: ATCTGAATTCCGTCCAGCTC & & & \\
\hline \multirow[t]{2}{*}{ SIFRG02 } & Solyc01g088040 & Unknown & F: GTTGATGAAGGGAGAGCCAT & 95 & 0.78 & 0.998 \\
\hline & & & R: GAGAGTAGATCACGGCAGCA & & & \\
\hline SIFRG03 & Solyc02g063070 & Unknown & F: GGCTGAACTGGCTCCTACTC & 109 & 0.77 & 0.999 \\
\hline SIFRG04 & & & R: TCAATACCACGAGCAAGGAG & & & \\
\hline \multirow[t]{2}{*}{ SIFRG05 } & Solyc01g104170 & Ankyrin repeat domain-containing protein & F: CAAAGGTTGATGCATTGGAC & 110 & 1.05 & 0.958 \\
\hline & & & R: AGTTACAGCGGCTCCATTCT & & & \\
\hline \multirow[t]{2}{*}{ SIFRG06 } & Solyc11g070030 & Unknown & F: CCACACGACACTTCCATCTC & 111 & 0.90 & 1 \\
\hline & & & R: TCAGCCGGTTTAGATATCCC & & & \\
\hline SIFRG08 & & & R: TTAATCATGTCTGCCACGGT & & & \\
\hline \multirow[t]{2}{*}{ SIFRGO9 } & Solyc03g031950 & unknown & F: CCCAАTTTCTTCCTCCGTAA & 108 & 0.98 & 0.954 \\
\hline & & & R: TGGCCTAAGAAGTCCGAATC & & & \\
\hline \multirow[t]{2}{*}{ SIFRG10 } & Solyc10g078450 & U6 snRNA-associated Sm-like protein & F: TATAGCAATGGAGCAAACCG & 112 & 0.75 & 0.997 \\
\hline & & & R: TGTCCTCTITGATGTGCTGA & & & \\
\hline \multirow[t]{2}{*}{ SIFRG11 } & Solyc05g023800 & Uncharacterized protein & F: ACAGGCACTGCATGAAGAAG & 109 & 0.91 & 0.993 \\
\hline & & & R: GTGAAACTGATCCGCTCTGA & & & \\
\hline \multirow[t]{2}{*}{ SIFRG12 } & Solyc04g009770 & Ran protein/TC4 protein 1 & F: AATCAAGTCCCAACCTGGAG & 93 & 0.90 & 0.989 \\
\hline & & & R: TTCATGAATGGCCTTTGGTA & & & \\
\hline \multirow[t]{2}{*}{ SIFRG13 } & Solyc01g103450 & DNAJ chaperone & F: AATGACAAGGTTTCCAAGGC & 100 & & \\
\hline & & & R: CTTCTAATCCAGCAATGCGA & & & \\
\hline \multirow[t]{2}{*}{ SIFRG17 } & Solyc04g056350 & Zinc finger family protein & F: ATGCAGCAACGACTAACAGG & 99 & 0.84 & 0.993 \\
\hline & & & R: TGCAAAGAGGACATTCAAGC & & & \\
\hline \multirow[t]{2}{*}{ SIFRG18 } & Solyc01g104700 & Ran protein/TC4 protein ran2b & F: ACTTAGCTGCACAGGCATTG & 125 & 0.91 & 0.989 \\
\hline & & & R: ATGGAAAGGTGCAACAGTGA & & & \\
\hline \multirow[t]{2}{*}{ SIFRG19 } & Solyc10g006480 & Ubiquitin & F: CAACCCTTCACTTGGTCCTT & 91 & 0.86 & 0.988 \\
\hline & & & R: CTTTCCACCTCAAGGGTGAT & & & \\
\hline \multirow[t]{2}{*}{ SIFRG20 } & Solyc10g083570 & Fructose-bisphosphatealdolase & F: TGTTGAGCCTGAGATCCTTG & 136 & 1.01 & 0.992 \\
\hline & & & R: GGGCTTCAACAATGTACCCT & & & \\
\hline \multirow[t]{2}{*}{ SIFRG21 } & Solyc02g089200 & SEP subfamily of the MADS-box gene $f$ & F: GGTGGTGAGCAAAGTCTCAA & 67 & 1.10 & 0.988 \\
\hline & & & R: GAGGTTGGAAGAAACCCTGA & & & \\
\hline SIFRG22 & Solyc08g081190 & plasma membrane intrinsic protein & F: GACCACTGGATCTTCTGGGT & 99 & 1.39 & 0.999 \\
\hline & & & R: TTAAGACCTGTGGAATGGCA & & & \\
\hline SIFRG23 & Solyc06g036540 & Unknown & F: GGGCTTATCAAGGAAGGGAT & 138 & 0.86 & 0.992 \\
\hline & & & R: GGCACCTCTTGTCACCTTI & & & \\
\hline SIFRG24 & Solyc02g080630 & Lactoylglutathionelyase & F: GGATGCAAGTGGTGAAGAAA & 144 & 1.03 & 0.996 \\
\hline & & & R: CGTATGCATTTCCCTTTGTG & & & \\
\hline SIFRG25 & Solyc05g050200 & Eukaryotic translation initiation factor $1 \mathrm{~A}$ & F: CGAAGAACAAGGGTAAGGGA & 137 & 0.90 & 0.993 \\
\hline
\end{tabular}


TABLE 2 | Continued

\begin{tabular}{|c|c|c|c|c|c|c|}
\hline Gene ID & Gene locus $^{a}$ & Gene description & Primer sequence $\left(5^{\prime}-3^{\prime}\right)$ & Product size (bp) & $\mathbf{E}$ & $R^{2}$ \\
\hline & & & R: ACATCGTCCATTACCAAGCA & & & \\
\hline \multirow[t]{2}{*}{ SIFRG26 } & Solyc01g087320 & tRNA-dihydrouridine synthase $A$ & F: TGTTGCTCCCATGATGAAAT & 97 & 0.94 & 0.994 \\
\hline & & & R: AGCAGCAAGCATTTCTGTGT & & & \\
\hline \multirow[t]{2}{*}{ SIFRG27 } & Solyc06g007510 & Ubiquitin-conjugating enzyme E2 & F: GCTCTCTGTTGACAGACCCA & 108 & 1.10 & 0.998 \\
\hline & & & R: GAGTCCAGCTACGAGCAGTG & & & \\
\hline \multirow[t]{2}{*}{ SIFRG29 } & Solyc02g084360 & V-type proton ATPase 16 kDaproteo lipid & F: TGCGCCTTCTTCGGTTCC & 88 & 0.94 & 0.993 \\
\hline & & & R: CACACCGCTCTTCGCTGTTC & & & \\
\hline \multirow[t]{2}{*}{ SIFRG30 } & Solyc09g082650 & Acireductonedioxygenase & F:GGCAAGAATATGTTGAGACGTTGTCA & 147 & 0.80 & 0.983 \\
\hline & & & R:CATCAAGAACAGCAAAGCCACCA & & & \\
\hline SIFRG31 & Solyc12g044600 & NADP-malic enzyme & F: TCAGCGCATATTGCTGCCAA & 125 & 0.90 & 0.996 \\
\hline \multirow[t]{2}{*}{ SIFRG33 } & Solyc12g055800 & Unknown & F: GCTTCAGACTTGGCCTGTACG & 127 & 0.91 & 1 \\
\hline & & & R: GGCACAAGTTCCACCAAGCA & & & \\
\hline \multirow[t]{2}{*}{ SIFRG34 } & Solyc12g008590 & Acireductonedioxygenase & F: TCGACGATCACCTCCTGTGTG & 81 & 1.12 & 0.962 \\
\hline & & & R: CCCAAACAGCGCCGTCAAG & & & \\
\hline \multirow[t]{2}{*}{ SIFRG35 } & Solyc02g085540 & NADH ubiquinone oxidoreductase & For: CGCCTGGACAGTCCCGTAAA & 127 & 0.87 & 0.998 \\
\hline & & & R: TGGGCCCAAGTTTCAAGGGT & & & \\
\hline \multirow[t]{2}{*}{ SIFRG36 } & Solyc09g009260 & fructose-1,6-bisphosphate aldolase & F: CTGAGTACACCGTCCGTGCT & 102 & 0.90 & 0.996 \\
\hline & & & R: GGTTGACGGTGGCTTCCTCT & & & \\
\hline \multirow[t]{2}{*}{ SIFRG37 } & Solyc11g005330 & Actin & F: GGAGATTGAAACTGCCAGGAGCA & 143 & 1.00 & 0.915 \\
\hline & & & R: CTGCAGCTTCCATACCAATCATGG & & & \\
\hline SIFRG38 & Solyc01g028810 & chaperonin & F:GTTGTGTGGTGGTTGAGATCAAGG & 74 & 0.99 & 0.915 \\
\hline
\end{tabular}

a Tomato gene ID in SGN (https://solgenomics.net/). E: Amplification efficiency; $R^{2}$ : Correlation coefficient.

analysis. Based on the previously reported RGs in tomato (Coker and Davies, 2003; Exposito-Rodriguez et al., 2008; Løvdal and Lillo, 2009; Mascia et al., 2010; Müller et al., 2015), 73 reported potential RGs of tomato were identified (Table 1). Moreover, by collecting the cDNA sequences of 11 previously reported candidate RGs for watermelon fruit study (From CuGenDB: Cucurbit genomics database, http://www.icugi.org/) (Kong et al., 2015), we subsequently collected their corresponding orthologs in tomato through blastN in the SGN (Table 1). Thus, a total of 84 tomato potential RGs were collected in the present study. Among all the reported RGs and their corresponding orthologs, three genes (Solyc01g028930, Solyc10g049850, and Solyc03g115810) were found to be redundant. Thus, a total of 81 previously reported RGs were collected and listed in Table $\mathbf{1}$.

According to the RNA-seq data derived from TFGD, RPKM values of the 81 previously reported RGs in five different fruit developmental stages $(1,2,3 \mathrm{~cm}$, MG [Mature green], B [Breaker], B10 [10 days after breaker]) of accession "Heinz" and in three developmental stages (IM [Immature], B [Breaker], B5 [5 days after breaker]) of accession "Pimp" were used to evaluate their expression stabilities (Supplementary Table 1). The CV values were calculated as described in Materials and Methods (Supplementary Table 3). Based on the RPKM values shown in Supplementary Table 1, we analyzed the relative expression of the 81 reported RGs among different developmental stages of tomato fruit. As shown in Supplementary Table 3, nearly 30\% $(24 / 81)$ of the 81 reported RGs demonstrated high expression variations $(\mathrm{CV}>0.35)$ during tomato fruit development (Figure 1A; Supplementary Table 3). Some CV values were even higher than 2.0 (Solyc12g096750/Solyc06g009860 [3.00], Solyc02g087880 [2.31]), suggesting their poor expression stabilities during tomato fruit development. Further analysis demonstrated that although the remaining 57 stably expressed reported RGs have CV values lower than 0.35 , most of them $(52 / 57)$ had the average expression levels (RPKM values) lower than 200, and some RPKM values of them (Solyc09g074220, Solyc12g096750, Solyc11g051210, and Solyc06g009860) were even close to 0 (low transcription level or undetected signal) (Supplementary Table 3), which indicated that these 52 reported RGs may not be qualified for normalization due to their low expression levels. According to Supplementary Table 3, only the PPKM values of five previously reported RGs, Solyc06g005060 
(1088.0), Solyc11g005330 (581.1), Solyc01g028810 (509.5), Solyc04g009770 (357.6), and Solyc05g023800 (359.9), were high enough $(>200)$ to be considered as candidate RGs in tomato fruit. Hence, we came to the conclusion that most of the previously reported candidate RGs identified so far were not well-qualified for normalization during tomato fruit development.

\section{Identification of Putative RGs Based on RNA-Seq Data Mining}

To comprehensively identify qualified RGs for tomato fruit, we searched the entire set of RNA-seq data derived from TFGD, and a total of 56 genes with RPKM values ranged between 200 and 2,000 among all developmental stages of tomato fruit were identified, by searching the derived RNA-seq data (Supplementary Table 2). The CV values of these genes were calculated, and more than $70 \%(40 / 56)$ of them were shown to be lower than 0.35 (Supplementary Table 3 ). The 40 genes were listed in details in Table 2. Further analysis revealed that the genes identified from the entire genome level (Figure 1B) were generally more stably expressed than those previously reported as candidate RGs (Figure 1A) during tomato fruit development.

\section{qPCR Analysis of the Putative RGs in Tomato Fruit Development Process}

Next, we intended to validate the expression stabilities of the selected 40 putative RGs by qPCR analysis. When designing the primers using genescript online tool (https://www.genscript. $\mathrm{com} /$ ), we found that proper primers of two candidate RGs (Solyc01g095050 [321 bp] and Solyc10g074860 [72 bp]) for qPCR analysis cannot be designed due to their short cDNA sequences or high homologies with other genes in tomato. Thus, a total of 38 candidate RGs, which are designated as SlFRG01 to SlFRG38, were eventually chosen for further expression validation in the qPCR experiments (Table 2).

qPCR amplification of the 38 candidate RGs were carried out using specific primers listed in Table 2, and the amplicon lengths ranged from $67 \mathrm{bp}$ (SlFRG21) to $149 \mathrm{bp}$ (SlFRG07). PCR-amplification specificity of each primer pair was verified by $1.5 \%$ agarose gel electrophoresis using cDNA templates (Supplementary Figure 1), and the melting curve analysis also showed single product peak (Supplementary Figure 2), which both confirm the specificities of the primer pairs. The amplification efficiencies (E) of these candidate RGs were found to vary from 0.76 (SlFRG01) to 1.39 (SlFRG22), and E values of more than $50 \%$ primer pairs (20/38) were ranged from 0.9 to 1.1 , indicating their qualifications as primer pairs (Tichopad et al., 2002; Chung et al., 2004). Notably, the amplification efficiencies (E) of SlFRG07 and SlFRG13 could not be calculated due to their low transcript level in tomato fruit (Supplementary Table 4). The correlation coefficients $\left(R^{2}\right)$ of the 38 candidate RGs ranged from 0.915 (SlFRG37/SlFRG38) to 1 (SlFRG06/SlFRG33) (Table 2).

The Ct values of the each putative RG derived from different tomato fruit developmental stages (Immature-IM; Mature Green-MG; Breaker-B; Mature Red-MR) (Figure 2;
Supplementary Table 4) were used here to evaluate the expression levels. The average $\mathrm{Ct}$ values of most candidate RGs $(36 / 38)$ in various fruit developmental samples ranged between 20 and 30 (Except for SlFRG07 [33.91] and SlFRG13 [32.29]) (Supplementary Table 4).

\section{Validation of Putative RGs Using geNorm and Normfinder}

Of all the 38 tested RGs, the geNorm analysis determined that the 12 most stable candidate RGs with average expression stabilities (M-value) less than 0.6, which were:SlFRG03, SlFRG27, SlFRG04, SlFRG23, SlFRG30, SlFRG24, SlFRG35, SlFRG37, SlFRG05, SlFRG16, SlFRG12, and SlFRG31 (Figure 3A; Table 3). To ensure the evaluation results, Normfinder was also conducted for evaluation, and 12 RGs with expression stabilities (M-value) less than 0.5 were identified, which were: SlFRG25, SlFRG35, SlFRG05, SlFRG14, SlFRG38, SlFRG17, SlFRG04, SlFRG37, SlFRG16, SlFRG27, SlFRG12, and SlFRG29 (Figure 3B; Table 3).

Although the two different assessing systems (geNorm and Normfinder) came up with different results, there were still seven putative RGs (SlFRG27, SlFRG04, SlFRG35, SlFRG05, SlFRG37, SlFRG16, SlFRG12) that were found to be commonly top-ranked in both statistical algorithms (Table 3). Generally speaking, the primer pairs with amplification efficiency (E) between 0.9 and 1.1 (Tiangen, China) possess the lowest variability in qPCR analysis (Tichopad et al., 2002; Chung et al., 2004). However, we found that among the seven top-ranked RGs, the amplification efficiencies (E) of two primer pairs (SlFRG04 [0.78] and SlFRG35 [0.87]) were lower than 0.9 , suggesting that the primer pairs of these two genes were not recommended for subsequent RG application in this study. Ct values of the remaining five RGs were all between 20 and 25 (qualified as RG). Therefore, SlFRG27, SlFRG05, SlFRG37, SlFRG16, and SlFRG12 were finally identified as qualified and optimal RGs for normalization in the tomato fruit developmental process.

Previously, some researchers have reported that the use of more than one internal control genes in normalization could effectively improve the reliability of qPCR results (Reid et al., 2006; Exposito-Rodriguez et al., 2008; Gutierrez et al., 2008). Thus, we applied the geNorm software to calculate the pairwise variation values $(\mathrm{V})$ of the 38 putative RGs (described in detailed in the Materials and Methods section). The pairwise variation revealed that the V2/3 value was 0.06 (significantly $<1.5$ ) (Figure 4), which indicated that the combined use of two most stably expressed RGs as reflected in geNorm, SlFRG03 and SlFRG27, was potentially sufficient for better normalization in $\mathrm{qPCR}$ experiments of tomato fruit developmental studies (Figure 4).

\section{DISCUSSION}

The advent of qPCR technology has brought a new revolution in the gene expression analysis area. Accurate interpretation of qRCR results mainly depends on the use of stable RGs for normalization, which can potentially minimize nonbiological variations of different samples. Hence, the systematic 


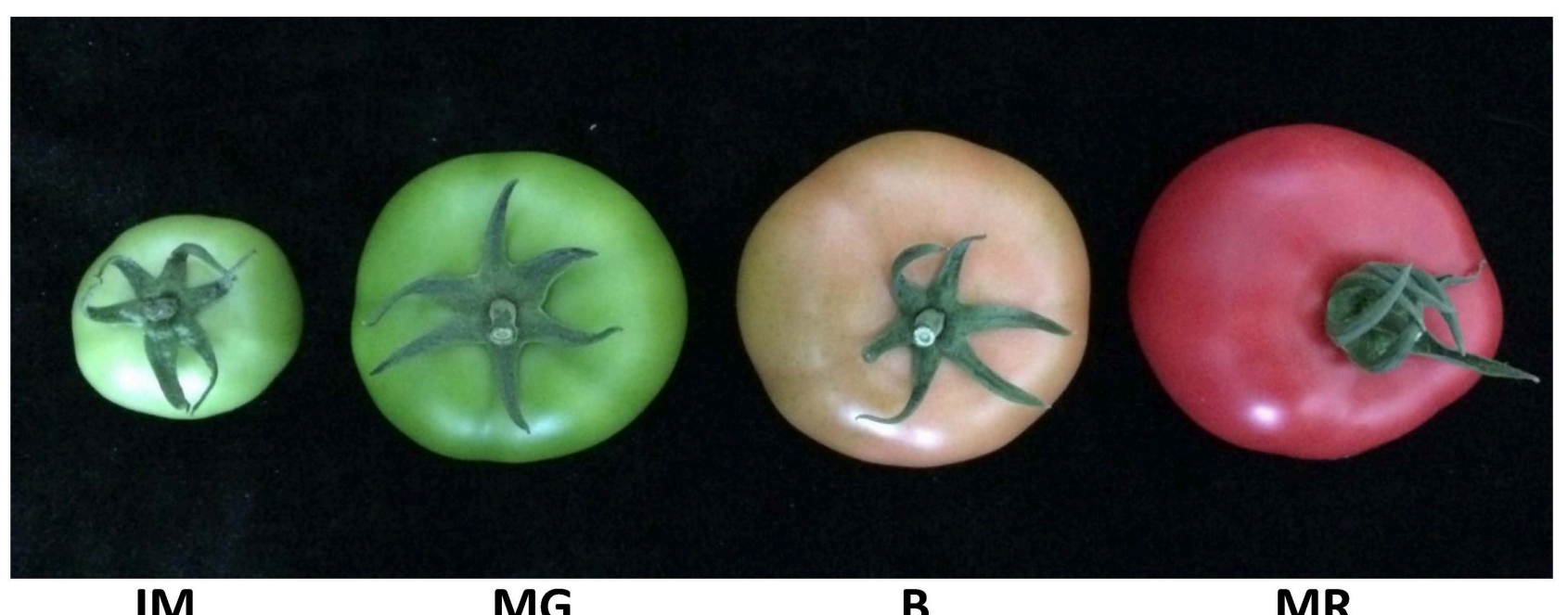

FIGURE 2 | Tomato fruit samples of four representative developmental stages. IM, Immature, 15 days after fertilization, $5 \mathrm{~cm}$ diameter fruit; MG, Mature green, 30 days after fertilization, $9 \mathrm{~cm}$ diameter fruit; B, Breaker, 35 days after fertilization, $10 \mathrm{~cm}$ diameter fruit; MR, Mature red, 45 days after fertilization, $10 \mathrm{~cm}$ diameter fruit.

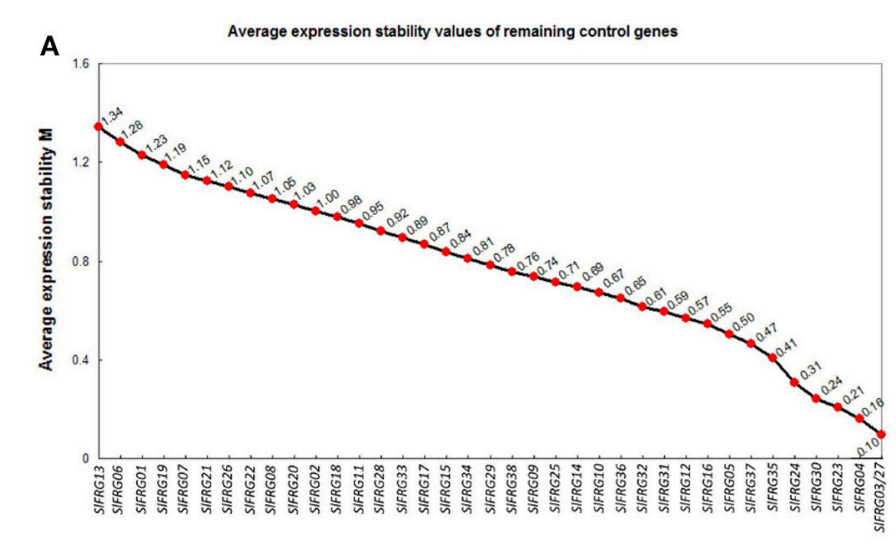

«::::: Least stable genes Most stable genes ::.::
B

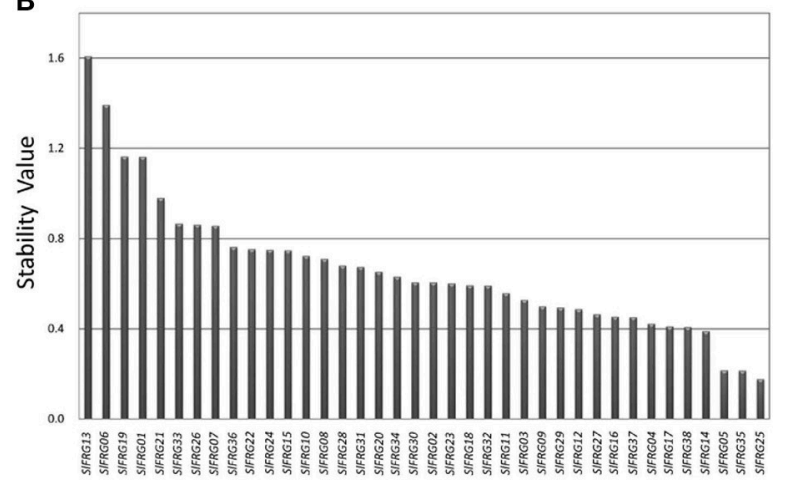

<::::: Least stable genes

Most stable genes :.:.:?

FIGURE 3 | Expression stability of the 38 newly identified RGs evaluated by geNorm (A) and NormFinder (B). (A) Ranking of geNorm is based on the principle that logarithmically transformed gene expression ratio between two ideal internal control genes should be identical if both genes are stably expressed in the tested sample set. Expression stability values (M) of the 38 candidate RGs are shown, RGs with a higher M value are less stably expressed. (B) NormFinder is a model-based approach that evaluates expression variation by comparing the variation within and between a certain number of sample groups and RGs with lower combined levels of intra and intra-group variation were regarded to be more stably expressed.

identification of appropriate RGs is essential for obtaining reliable results in qPCR experiments (Udvardi et al., 2008; Bustin et al., 2009; Guenin et al., 2009). Nowadays, some HKGs (e.g., Actin, Ubiquitin, and 18s rRNA), are usually used as RGs under various experimental conditions, or across a broad range of tissue samples (Bustin, 2002; Kong et al., 2014). However, an increasing number of evidence showed that optimal RGs varied depending on the experimental conditions or organs/tissues assayed, and it seems to be impossible to acquire a list of RGs universally practicable across a wide range of experimental conditions (Guenin et al., 2009; Warzybok and Migocka, 2013; Kong et al., 2014). Therefore, the identification of suitable RGs for specific experimental conditions is essential for avoiding unnecessary error in the qRCR experimental results. So far, many studies involving the identification or evaluation of tomato RGs under various experimental conditions, including biotic/abiotic stresses (Cucumber mosaic virus, tobacco mosaic virus, bacterium Xanthomonas campestris, nitrogen stress, cold, light stress) (Coker and Davies, 2003; Løvdal and Lillo, 2009; Mascia et al., 2010; Wieczorek et al., 2013; Müller et al., 2015) and different organs/tissues (Leaf, fruit, flower, and seed) (ExpositoRodriguez et al., 2008; Dekkers et al., 2012; Gao et al., 2012; Baldassarre et al., 2015), had been conducted. For example, SlACT, SlCAC, and SlEF1 $\alpha$ were validated to be suitable RGs in 
TABLE 3 | Top-ranked RGs evaluated by geNorm and NormFinder.

\begin{tabular}{lclcl}
\hline geNorm & M-value & NormFinder & M-value & Consensus \\
\hline SIFRG03 & 0.095 & SIFRG25 & 0.177 & SIFRG27 \\
SIFRG27 & 0.095 & SIFRG35 & 0.215 & SIFRG04 \\
SIFRG04 & 0.156 & SIFRG05 & 0.216 & SIFRG35 \\
SIFRG23 & 0.206 & SIFRG14 & 0.390 & SIFRG05 \\
SIFRG30 & 0.242 & SIFRG38 & 0.408 & SIFRG37 \\
SIFRG24 & 0.305 & SIFRG17 & 0.411 & SIFRG16 \\
SIFRG35 & 0.409 & SIFRG04 & 0.423 & SIFRG12 \\
SIFRG37 & 0.467 & SIFRG37 & 0.452 & \\
SIFRG05 & 0.504 & SIFRG16 & 0.454 & \\
SIFRG16 & 0.547 & SIFRG27 & 0.464 & \\
SIFRG12 & 0.569 & SIFRG12 & 0.487 & \\
SIFRG31 & 0.594 & SIFRG29 & 0.494 & \\
\hline
\end{tabular}

The seven RGs identified commonly in geNorm and Normfinder were labeled in bold.

studies of host-virus interactions in tomato (Wieczorek et al., 2013). Exposito-Rodriguez et al. (2008) found that the widely used RGs, such as SICAC, SITIP41, Expressed, and SISAND, provide superior transcript normalization in various tissues of tomato (Exposito-Rodriguez et al., 2008). When studying the changes of gene expression in the wounded ripening-stage tomato fruit, Baldassarre et al. (2015) selected two most stably expressed RGs (EF1- $\alpha$ and GADPH) from seven routine used HKGs for normalization in the subsequent qPCR analysis. Thus, it occurred to us that although tomato is regarded as model plant for fruit development study, little attention has yet been paid to screen the best RGs specifically for normalization during the development of tomato fruit.

So far, most studies involving RG identification were based on the evaluation or validation of the expression stabilities of traditional or novel RGs under corresponding conditions (Czechowski et al., 2005; Løvdal and Lillo, 2009; Schmidt and Delaney, 2010; Dekkers et al., 2012; Baldassarre et al., 2015). In the present study, we collected 70 putative RGs that had been previously reported in tomato (Coker and Davies, 2003; Exposito-Rodriguez et al., 2008; Løvdal and Lillo, 2009; Mascia et al., 2010; Müller et al., 2015) and 11 orthologs of reported RGs in watermelon fruit study (Kong et al., 2015), and subsequently validated their expression stabilities during different stages of fruit development according to the RPKM values derived from RNA-seq data sets. Out of expectation, the majority of these putative RGs identified previously were not well-qualified for normalization as internal control genes due to either their unstable expressions or low transcript levels (Table 1; Figure 1A; Supplementary Table 1). Therefore, we next intended to identify some novel RGs that are stably expressed during the whole developmental process of tomato fruit.

Nowadays, the open sources of the SGN and TFGD allowed us to search for the most stably expressed genes on a comprehensive evaluation system. In the present study, we conducted a data mining based on the RNA-seq data set of different tomato fruit developmental stages, and initially collected 38 most stably expressed genes as putative RGs for normalization in tomato fruit developmental study (Table 2). Further evaluation of these 38 putative RGs was conducted using qPCR analysis in four developmental stages of tomato fruit (IM, MG, B, and $M G$ ) (Figure 2). Next, we used two popular statistical algorithms for RG ranking, geNorm, and Normfiner (Vandesompele et al., 2002; Andersen et al., 2004), for the RG evaluation based on the qPCR results (Supplementary Table 4). We found different ranking results from the evaluation results of geNorm and Normfinder (Table 3), which were explicable as these two algorithms are based on different models and assumptions (Schmidt and Delaney, 2010). The geNorm algorithm is based on the principle that logarithmically transformed expression ratio of two genes should be constant if both of them are stably expressed in the tested sample set. The relative stability of each gene $(M)$ is defined as the mean pairwise variation (reflected by standard deviation of the expression ratios of two genes) of the gene in the sample set. Furthermore, as normalization with single RG can cause inevitable errors, geNorm is also used to determine the minimum number of RGs required for more reliable normalization (Vandesompele et al., 2002). Normfinder measures gene expression stability by comparing the variation within and between a certain number of sample groups. The genes with the lowest combined levels of intra and inter-group variation were regarded as most stably expressed (Andersen et al., 2004; Schmidt and Delaney, 2010). Taken together, Normfinder is based on analyzing the variation level of each tested gene rather than pairwise analysis of gene stability relative to a set of potential RGs (Schmidt and Delaney, 2010). So far, numerous ranking differences of RGs derived by these two algorithms had been found in many previous studies (Schmidt and Delaney, 2010; Cassan-Wang et al., 2012; Kong et al., 2015). Nevertheless, we identified 7 putative RGs (SlFRG04, SlFRG35,SlFRG27, SlFRG05, SlFRG37, SlFRG16, and SlFRG12) that were common in geNorm and Normfinder. Considering the unqualified primer amplification efficiencies (E) of SlFRG04 and SlFRG35, these two genes were excluded from our recommendation list, and the remaining five genes (SlFRG05, SlFRG12, SlFRG16, SlFRG27, and SlFRG37) were finally identified as suitable internal controls for normalization in tomato fruit development. Notably, we believe that some alternative primer pairs of SlFRG04 and SlFRG35 with improved amplification efficiencies might be redesigned for RG use in tomato fruit developmental study.

In practice, it is believed that the use of more than one RG in the normalization can efficiently improve the reliability of qPCR results (Alba and Giovannoni, 2005; Exposito-Rodriguez et al., 2008; Gutierrez et al., 2008). Thus, in order to explore the minimun number of RGs needed, the pairwise variation (V) values were calculated in geNorm (Figure 4). According to the evaluation, the combined application of two RGs, SlFRG03, and SlFRG27, would be a better choice than the use of only one RG for normalization when more reliable $\mathrm{qPCR}$ results are needed. It is also worth noting that due to the multiple sections of tomato fruit and the complex biological processes of fruit development, gene expression analysis has been extended to more precise tissue parts (e.g., pericarp, flesh, and even seeds) or longer developmental stages of fruits (Fraser et al., 1994; Carrari and Fernie, 2006; 
Determination of the optimal number of control genes for normalization

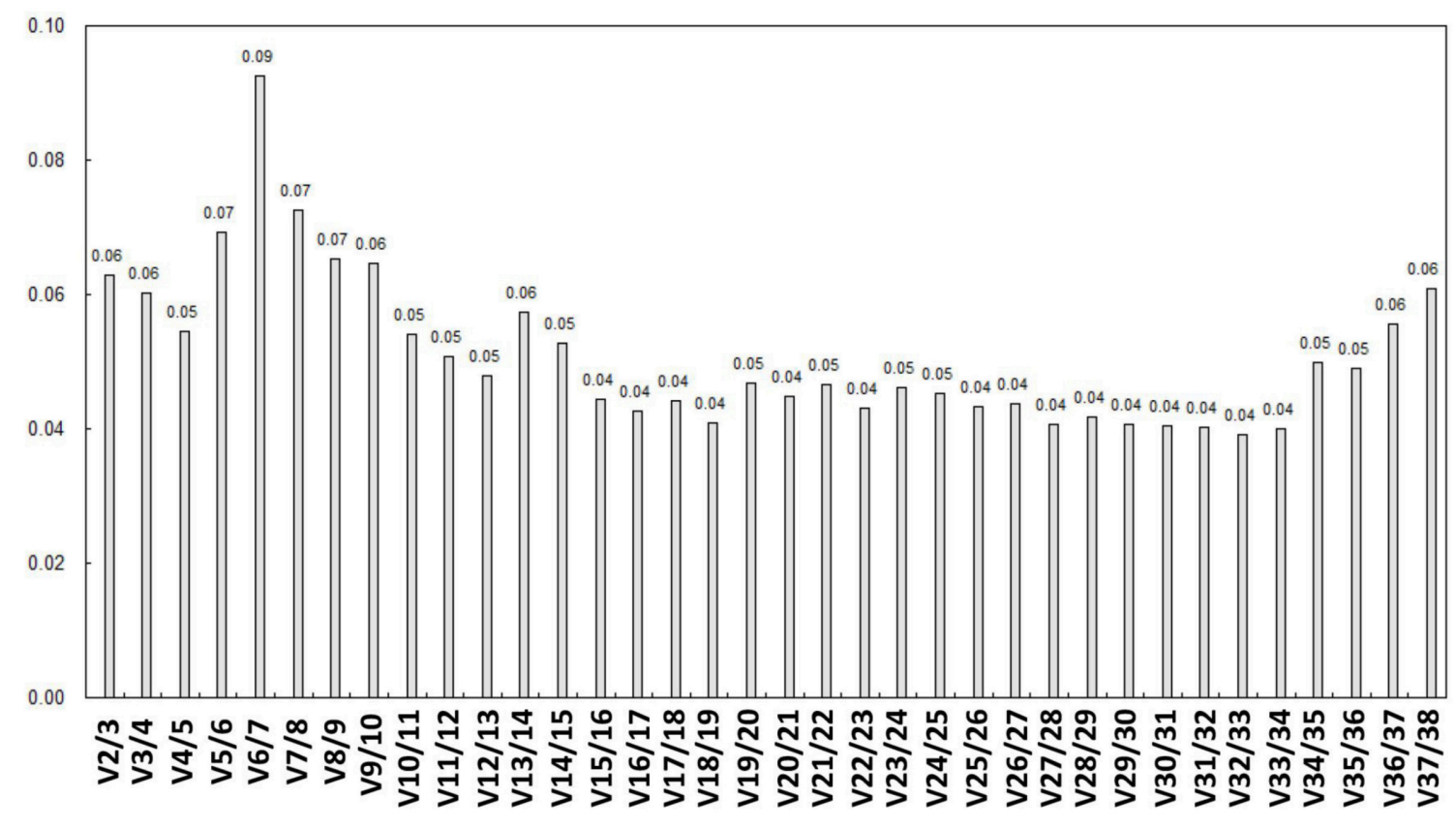

Pairwise Variations

FIGURE 4 | Analysis of best RG association based on geNorm algorithm. The optimum number of RGs is the lowest number of genes with an acceptably low $V n / n+1$. Vandesompele et al. (2002) suggested 0.15 (15\% variation in normalization factors) to be an upper limit for $\mathrm{Vn} / \mathrm{n}+1$. According to variations ( $\mathrm{V}$-value) calculation, V2/3 is $0.06(<0.15)$, which means the most stable expressed RGs identified ingeNorm, SIFRG03 and SIFRG27, are well-qualified as RG combination for normalization.

Fei et al., 2011; Cheng et al., 2016). Therefore, we propose that the RGs identified in this study should be further validated in different tissue sections or earlier developmental stages (e.g., 1, 2 $\mathrm{cm}$ green fruits) of tomato fruit in the future according to specific experimental requirements.

\section{CONCLUSION}

To our knowledge, this study is the first systematic identification and evaluation of putative RGs as internal controls for normalization of qPCR analysis in tomato fruit developmental process. According to our extensive evaluation, five identified RGs-SlFRG05, SlFRG12, SlFRG16, SlFRG27, and SlFRG37 could be recommended for normalization of $\mathrm{qPCR}$ experiments in tomato fruits. Furthermore, according to geNorm analysis, a combination of two most stably expressed RGs, SlFRG03 and SlFRG27, were recommended when more reliable qPCR results were needed. Moreover, by comparative analysis of the previously published materials involving RG identification for fruit developmental study in other plants, we found that two RGs identified in this study were also chosen as optimal RGs for fruit developmental study in other plants (Zhu et al., 2012; Die and Rowland, 2013; Kong et al., 2015), which are ubiquitin conjugating enzyme (UBI) encoding genes (SlFRG27 in tomato, PEX4 and UBC28 in blueberry, UBCE in papaya) and actin encoding genes (SlFRG37 in tomato, ClACT in watermelon, ACTIN in papaya). Thus, SlFRG27/SlFRG37 and their corresponding orthologs seem to be universally applicable as RGs among plants of different families including Cucurbitaceae, Rosaceae, Vacciniaceae, and Solanacea. Taken together, the results presented here not only unveil optimal RGs for qPCR analysis in tomato fruit development, but also provide referable guidelines for identification of RGs in other plant species.

\section{AUTHOR CONTRIBUTIONS}

Conceived and designed the experiments: $\mathrm{HW}$ and $\mathrm{YC}$; Performed the experiments: YC, WB, XP, JY, MR, QY, RW, ZL, GZ, and ZY; Analyzed the data: JY, WB, XP and GZ; Wrote the paper: YC, WB, XP, GA, and HW. All authors have read and approved the manuscript.

\section{ACKNOWLEDGMENTS}

This research was supported by the National Natural Science Foundation of China (31501749, 31301774, 31272156, 31772294), the Zhejiang Provincial Natural Science Foundation of China (LQ15C150002), Jiangsu Provincial Natural Science Foundation of China (BK20140277), Suzhou Science and 
Technology Project (SYN201418), the Zhejiang Provincial Agricultural (Vegetable) New Variety Breeding Project (2016C02051-2-3, 2016C02051-1-1, 2016C02051-1-3), the Public Agricultural Technology Research in Zhejiang (2016C32101, 2015C32049), and the Technological System of Ordinary Vegetable Industry (CARS-25-G-16).

\section{SUPPLEMENTARY MATERIAL}

The Supplementary Material for this article can be found online at: http://journal.frontiersin.org/article/10.3389/fpls.2017. 01440/full\#supplementary-material

Supplementary Figure 1 | PCR amplification products of the newly identified RGs on cDNA templates.

\section{REFERENCES}

Alba, R., and Giovannoni, J. J. (2005). Transcriptome and selected metabolite analyses reveal multiple points of ethylene control during tomato fruit development. Plant Cell 17, 2954-2956. doi: 10.1105/tpc.105.036053

Andersen, C. L., Jensen, T. F., and Orntoft, T. F. (2004). Normalization of real-time quantitative reverse transcription-PCR data: a model-based variance estimation approach to identify genes suited for normalization, applied to bladder and colon cancer data sets. Cancer Res. 64, 5245-5250. doi: 10.1158/0008-5472.CAN-04-0496

Baldassarre, V., Cabassi, G., Spadafora, N. D., Aprile, A., Müller, C. T., Rogers, H. J., et al. (2015). Wounding tomato fruit elicits ripening-stage specific changes in gene expression and production of volatile compounds. J. Exp. Bot. 66, 1511-1526. doi: 10.1093/jxb/eru516

Bustin, S. A. (2002). Quantification of mRNA using real-time reverse transcription PCR (RT-PCR): trends and problems. J. Mol. Endocrinol. 29, 23-29. doi: $10.1677 /$ jme.0.0290023

Bustin, S. A., Benes, V., Garson, J. A., Hellemans, J., Huggett, J., and Kubista, M. (2009). The MIQE Guidelines: minimum information for publication of quantitative real-time PCR experiments. Clin. Chem. 55, 611-622. doi: 10.1373/clinchem.2008.112797

Bustin, S. A., Benes, V., Nolan, T., and Pfaffl, M. W. (2005). Quantitative realtime RT-PCR-a perspective. J. Mol. Endocrinol. 34, 597-601. doi: 10.1677/jme.1.01 755

Bustin, S. A., and Nolan, T. (2004). Pitfalls of quantitative real-time reversetranscription polymerase chain reaction. J. Biomol Tech. 15, 155-166.

Carrari, F., and Fernie, A. R. (2006). Metabolic regulation underlying tomato fruit development. J. Exp. Bot. 57, 1883-1897. doi: 10.1093/jxb/erj020

Cassan-Wang, H., Soler, M., Camargo, E. L., Carocha, V., Ladouce, N., and Savelli, B. (2012). Reference genes for high-throughput quantitative reverse transcription-PCR analysis of gene expression in organs and tissues of Eucalyptus grown in various environmental conditions. Plant Cell Physiol. 53, 101-116. doi: 10.1093/pcp/pcs152

Cheng, Y., Jalal, A. G., Yu, J., Yao, Z., Ruan, M., and Ye, Q. (2016). Putative WRKYs associated with regulation of fruit ripening revealed by detailed expression analysis of the WRKY gene family in pepper. Sci. Rep. 6:39000. doi: $10.1038 /$ srep39000

Chung, D. T., Drabek, J., Opel, K. L., Butler, J. M., and Mccord, B. R. (2004). A study on the effects of degradation and template concentration on the amplification efficiency of the STR miniplex primer sets. J. Forensic Sci. 49, 733-740. doi: 10.1520/JFS2003269

Coker, J. S., and Davies, D. (2003). Selection of candidate housekeeping controls in tomato plants using EST data. Biotechniques 35, 740-742.

Colombiet, S., Beauvoit, B., Nazaret, C., Benard, C., and Vercambre, G. (2016). Respiration climacteric in tomato fruits elucidated by constraint-based modelling. New Phytol. 213, 1726-1739. doi: 10.1111/nph.14301

Czechowski, T., Stitt, M., Altmann, T., Udvardi, M. K., and Scheible, W. R. (2005). Genome-wide identification and testing of superior reference
Supplementary Figure 2 | Specificity of the newly identified RG primers demonstrated in GPCR analysis. Melting curves generated for all amplicons.

Supplementary Table 1 | Expression levels (RPKM-value) of previoulsly reported RGs against RNA-seq data of different developmental stages of tomato fruit.

Supplementary Table 2 | Expression levels (RPKM-value) of newly identified RGs against RNA-seq data of different developmental stages of tomato fruit.

Supplementary Table 3 | CV (Co-efficient variation), average expression (AVE) and standard deviation (SD) values of previous reported RGs and newly identified RGs in tomato.

Supplementary Table 4 | Ct-values of 38 newly identified RGs during tomato fruit development (IM, MG, B, MR).

Supplementary Table 5 | Expression stability (M) values of 38 newly identified RGs evaluated by geNorm and NormFinder.

genes for transcript normalization in arabidopsis. Plant Physiol. 139, 5-17. doi: $10.1104 /$ pp.105.063743

Dekkers, B. J., Willems, L., Bassel, G. W., van Bolderen-Veldkamp, R. P., Ligterink, W., and Hilhorst, H. W. (2012). Identification of reference genes for RT-qPCR expression analysis in arabidopsis and tomato seeds. Plant Cell Physiol. 53, 28-37. doi: 10.1093/pcp/pcr113

Die, J. V., and Rowland, L. J. (2013). Superior cross-species reference genes: a blueberry case study. PLOS ONE 8:e73354. doi: 10.1371/journal.pone. 0073354

Exposito-Rodriguez, M., Borges, A. A., Borges-Perez, A., and Perez, J. A (2008). Selection of internal control genes for quantitative real-time RTPCR studies during tomato development process. BMC Plant Biol. 8:131. doi: 10.1186/1471-2229-8-131

Fei, Z., Joung, J. G., Tang, X., Zheng, Y., Huang, M., and Lee, J. M. (2011) Tomato functional genomics database: a comprehensive resource and analysis package for tomato functional genomics. Nucleic Acids Res. 39, D1156-D116. doi: 10.1093/nar/gkq991

Fraser, P. D., Truesdale, M. R., Bird, C. R., Schuch, W., and Bramley, P. M. (1994). Carotenoid biosynthesis during tomato fruit development (evidence for tissue-specific gene expression). Plant Physiol. 105, 405-413. doi: 10.1104/pp.105.1.405

Gachon, C., Mingam, A., and Charrier, B. (2004). Real-time PCR: what relevance to plant studies. J. Exp. Bot. 55, 1445-1454. doi: 10.1093/jxb/ erh 181

Gao, S., Xu, T., Qi, M., Liu, Y., Li, H., and Lv, S. (2012). Evaluation of the expression of internal control transcripts by real-time RT-PCR analysis during tomato flower abscission. Afr. J. Biotechnol. 11, 12983-12989. doi: 10.5897/AJB12.931

Ginzinger, D. G. (2002). Gene quantification using real-time quantitative PCR: an emerging technology hits the mainstream. Exp. Hematol. 30, 503-512. doi: 10.1016/S0301-472X(02)00806-8

Guenin, S., Mauriat, M., Pelloux, J., Van Wuytswinkel, O., Bellini, C., and Gutierrez, L. (2009). Normalization of qRT-PCR data: the necessity of adopting a systematic, experimental conditions-specific, validation of references. J. Exp. Bot. 60, 487-493. doi: 10.1093/jxb/ern305

Gutierrez, L., Mauriat, M., Pelloux, J., Bellini, C., and Van Wuytswinkel, O. (2008). Towards a systematic validation of references in real-time RT-PCR. Plant Cell 20, 1734-1735. doi: 10.1105/tpc.108.059774

Huggett, J., Dheda, K., Bustin, S., and Zumla, A. (2005). Real-time RT-PCR normalisation: strategies and considerations. Genes Immun. 6, 279-284. doi: $10.1038 /$ sj.gene. 6364190

Jacob, F., Guertler, R., Naim, S., Nixdorf, S., Fedier, A., and Hacker, N. F. (2013). Careful selection of reference genes is required for reliable performance of RT-qPCR in human normal and cancer cell lines. PLoS ONE 8:e59180. doi: 10.1371/journal.pone.0059180

Jain, M., Nijhawan, A., Tyagi, A. K., and Khurana, J. P. (2006). Validation of housekeeping genes as internal control for studying gene expression in rice by quantitative real-time PCR. Biochem. Biophys. Res. Commun. 345, 646-651. doi: 10.1016/j.bbrc.2006.04.140 
Jarosova, J., and Kundu, J. (2010). Validation of reference genes as internal control for studying viral infections in cereals by quantitative real-time RT-PCR. BMC Plant Biol. 10:146. doi: 10.1186/1471-2229-10-146

Jian, B., Liu, B., Hou, W., Wu, C., and Han, T. (2008). Validation of internal control for gene expression study in soybean by quantitative real-time PCR. BMC Mol. Biol. 9:59. doi: 10.1186/1471-2199-9-59

Klee, H. J., and Giovannoni, J. J. (2011). Genetics and control of tomato fruit ripening and quality attributes. Annu. Rev. Genet. 45, 41-59. doi: 10.1146/annurev-genet-110410-132507

Kong, Q. S., Yuan, J., Gao, L., Zhao, L., Cheng, F., and Huang, Y. (2015). Evaluation of appropriate reference genes for gene expression normalization during watermelon fruit development. PLOS ONE 10:e130865. doi: 10.1371/journal.pone.0130865

Kong, Q., Yuan, J., Niu, P., Xie, J., Jiang, W., and Huang, Y. (2014). Screening suitable reference genes for normalization in reverse transcription quantitative real-time PCR analysis in melon. PLoS ONE 9:e87197. doi: 10.1371/journal.pone.0087197

Løvdal, T., and Lillo, C. (2009). Reference gene selection for quantitative real-time PCR normalization in tomato subjected to nitrogen, cold, and light stress. Anal. Biochem. 387, 238-242. doi: 10.1016/j.ab.2009.01.024

Mascia, T., Santovito, E., Gallitelli, D., and Cillo, F. (2010). Evaluation of reference genes for quantitative reverse-transcription polymerase chain reaction normalization in infected tomato plants. Mol. Plant Pathol. 11, 805-816. doi: 10.1111/j.1364-3703.2010.00646.x

Müller, O. A., Grau, J., Thieme, S., Prochaska, H., Adlung, N., and Sorgatz, A. (2015). Genome-wide identification and validation of reference genes in infected tomato leaves for quantitative RT-PCR analyses. PLoS ONE 10:e0136499. doi: 10.1371/journal.pone.0136499

Pfaffl, M. W. (2004). “Quantification strategies in real-time PCR," in $A Z$ of quantitative PCR, ed S. A. Bustin (La Jolla, CA: International University Line), 87-112.

Reid, K. E., James, S., Niclas, O., Reid, K. E., and Lund, S. T. (2006). An optimized grapevine RNA isolation procedure and statistical determination of reference genes for real-time RT-PCR during berry development. BMC Plant Biol. 6:27. doi: 10.1186/1471-2229-6-27

Ruiz-May, E., and Rose, J. K. (2012). Progress toward the tomato fruit cell wall proteome. Front. Plant Sci. 4:159. doi: 10.3389/fpls.2013.00159

Schmidt, G. W., and Delaney, S. K. (2010). Stable internal reference genes for normalization of real-time RT-PCR in tobacco (Nicotiana tabacum) during development and abiotic stress. Mol. Genet. Genomics 283, 233-241. doi: 10.1007/s00438-010-0511-1

Stürzenbaum, S., and Kille, P. (2001). Control genes in quantitative molecular biological techniques: the variability of invariance. Comp. Biochem. Physiol. $B$ Biochem. Mol. Biol. 130, 281-289. doi: 10.1016/S1096-4959(01)00440-7

Suzuki, T., Higgins, P. J., and Crawford, D. R. (2000). Control selection for RNA quantitation. Biotechniques 29, 332-337.
Tichopad, A., Dzidic, A., and Pfaffl, M. W. (2002). Improving quantitative realtime RT-PCR reproducibility by boosting primer-linked amplification efficiency. Biotechnol. Lett. 24, 2053-2056. doi: 10.1023/A:10213194 21153

Tieman, D., Zhu, G., Resende, M. F., Lin, T., Nguyen, C., and Bies, D. (2017). A chemical genetic roadmap to improved tomato flavor. Science 27, 391-394. doi: $10.1126 /$ science.aal1556

Udvardi, M. K., Czechowski, T., and Scheible, W. R. (2008). Eleven golden rules of quantitative RT-PCR. Plant Cell 20, 1736-1737. doi: 10.1105/tpc.108.0 61143

Vandesompele, J., de Preter, K., Pattyn, F., Poppe, B., van Roy, N., dePaepe, A., et al. (2002). Accurate normalization of real-time quantitative RT-PCR data by geometric averaging of multiple internalcontrol genes. Genome Biol. 3:research0034.1. doi: 10.1186/gb-2002-3-7-research0034

Wang, S. B., Liu, K. W., Diao, W. P., Zhi, L., Ge, W., and Liu, J. B. (2012). Evaluation of appropriate reference genes for gene expression studies in pepper by quantitative real-time PCR. Mol. Breed. 30, 1393-1400. doi: $10.1007 / \mathrm{s} 11032-012-9726-7$

Warzybok, A., and Migocka, M. (2013). Reliable reference genes for normalization of gene expression in cucumber grown under different nitrogen nutrition. PLOS ONE 8:e72887. doi: 10.1371/journal.pone.00 72887

Wieczorek, P.,Wrzesińska, B., and Obrępalska-Stęplowska, A. (2013). Assessment of reference gene stability influenced by extremely divergent disease symptoms in Solanum lycopersicum L. J. Virol. Methods 194, 161-168. doi: 10.1016/j.jviromet.2013.08.010

Yoo, W. G., Kim, T. I., Li, S., Kwon, O. S., Cho, P. Y., and Kim, T. S. (2009). Reference genes for quantitative analysis on Clonorchissinensis gene expression by real-time PCR. Parasitol. Res. 104, 321-328. doi: 10.1007/s00436-0081195-x

Zhu, X., Li, X. P., Chen, W. X., Chen, J. Y., Lu, W. J., and Chen, L. (2012). Evaluation of new reference genes in papaya for accurate transcript normalization under different experimental conditions. PLoS ONE 7:e44405. doi: 10.1371/journal.pone.0044405

Conflict of Interest Statement: The authors declare that the research was conducted in the absence of any commercial or financial relationships that could be construed as a potential conflict of interest.

Copyright (c) 2017 Cheng, Bian, Pang, Yu, Ahammed, Zhou, Wang, Ruan, Li, Ye, Yao, Yang and Wan. This is an open-access article distributed under the terms of the Creative Commons Attribution License (CC BY). The use, distribution or reproduction in other forums is permitted, provided the original author(s) or licensor are credited and that the original publication in this journal is cited, in accordance with accepted academic practice. No use, distribution or reproduction is permitted which does not comply with these terms. 